\title{
Contrast transesophageal three-dimensional echocardiographic imaging for patent foramen ovale: a needful role?
}

\author{
Reem Al Sergani' ${ }^{1}$, Sara Di Michele ${ }^{2}$, Abdulhalim J. Kinsara ${ }^{3}$, Domenico Galzerano ${ }^{4,5}$, Olga Vriz ${ }^{4,5}$, \\ Isra Elmahi ${ }^{5}$, Antonio Cittadini ${ }^{6}$, Paolo Colonna ${ }^{7}$, Khaled M. AlKattan ${ }^{4,5}$ \\ ${ }^{1}$ College of Medicine, King Saud University, Riyadh, Saudi Arabia; ${ }^{2}$ San Filippo Neri Hospital, Cardiology Division, \\ Rome, Italy; ${ }^{3}$ Ministry of National Guard Health Affair, King Saud Bin Abdulaziz University for Health Sciences, \\ COM-WR, King Abdullah International Research Center, Jeddah, Saudi Arabia; ${ }^{4}$ The Heart Centre, King Faisal \\ Specialist Hospital \& Research Centre, Riyadh, Saudi Arabia; ${ }^{5}$ College of Medicine, Alfaisal University, Riyadh, Saudi \\ Arabia; ${ }^{6}$ Department of Clinical and Surgical Medicine, Federico II University, Napoli, Italy; ${ }^{7}$ Ecocardiografia Basale \\ e Complessa, Azienda Ospedaliera Universitaria, Policlinico di Bari, Italy
}

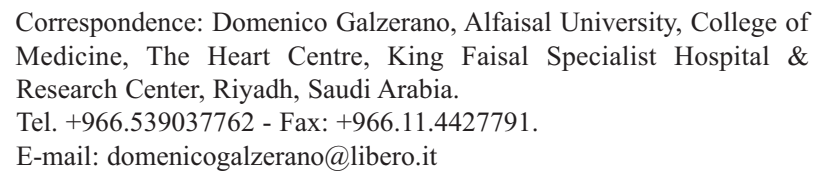

Key words: Patent foramen ovale; transesophageal echocardiography; three-dimensional echocardiography.

Acknowledgments: We would like to thank Abdul Rahim, RCS, for the master collaboration in performing the studies and in analyzing and reviewing the images and the results. We also thank Susanna Wright for the English language editing.

Authors' contributions: All the authors equally contributed to this work. RAS, began the project, wrote, reviewed the manuscript and the figures, looked for and carefully examined the references and approved the final manuscript; SDM, interpreted and commented echocardiographic imaging, wrote, reviewed and approved the final manuscript; AJK, interpreted and commented echocardiographic imaging, wrote, reviewed and approved the final manuscript; DG, OV, collected clinical data, performed, interpreted and commented echocardiographic imaging, wrote, reviewed and approved the final manuscript; IE, collected clinical data, wrote, reviewed the manuscript and the figures, looked for and carefully examined the references and approved the final manuscript; AC, PC, interpreted and commented echocardiographic imaging, wrote, reviewed and approved the final manuscript; KMA, interpreted and commented clinical results, wrote, reviewed and approved the final manuscript. All the authors have read and approved the final version of the manuscript and agreed to be accountable for all aspects of the work.

Conflict of interest: The authors declare that they have no competing interests, and all authors confirm accuracy.

Informed consent: obtained from a legally authorized representative for anonymized patient information to be published in this article.

Received for publication: 20 March 2020.

Accepted for publication: 25 May 2020.

${ }^{\circ}$ Copyright: the Author(s), 2020

Licensee PAGEPress, Italy

Monaldi Archives for Chest Disease 2020; 90:1278

doi: $10.4081 /$ monaldi.2020.1278

This article is distributed under the terms of the Creative Commons Attribution Noncommercial License (by-nc 4.0) which permits any noncommercial use, distribution, and reproduction in any medium, provided the original author(s) and source are credited.

\section{Abstract}

The management of patent foramen ovale (PFO) still remains controversial. According to the newest scientific evidences, the assessment of the PFO size and the shunt has paramount importance for further management [1]. Although two-dimensional (2D) transesophageal (TE) echocardiography (E) contrast study has been considered to be the gold standard imaging modality for diagnosing PFO for a long time, new evidences underscore that its accuracy may have been over-valued [1,2]. Three-dimensional (3D) TEE imaging has emerged as a diagnostic technique that overcome some of the limitations of 2DE. 3D TEE allows an anatomical imaging for a comprehensive echocardiographic assessment of the left atrium, atrial septum and of PFO morphology $[3,4]$. However, it has never been reported about its diagnostic usefulness in detecting the bubbles passage during contrast study.

We report a case where it has been possible to detect the passage of bubbles across the PFO thanks to 3D TEE.

\section{Case Report}

We report a case of a 55-year-old male admitted for cardiogenic embolic ischemic stroke work up including transthoracic (TT) and TEE contrast study. PFO was defined as TT and TE echocardiographic evidence of infused microbubbles in the left atrium within three cardiac cycles after their appearance in the right atrium, at rest or during Valsalva release; echo contrast consisted of an agitated mixture of air, saline solution and patient's blood; the ability of the patient to correctly perform the Valsalva manoeuvre to provoke a right-to-left shunt was assessed by observing a $20 \mathrm{~cm} / \mathrm{s}$ decrease in transmitral E velocity by TT echocardiography before the TEE contrast study and/or a change in the convexity of the fossa ovalis membrane from right to left. Transthoracic echocardiography did not reveal any sources of embolism and passage of bubbles during contrast study. Further 2D TEE with contrast study to rule PFO and sources of embolism was performed ; 2D TEE analysis did not detect any bubbles passage in the left atrium at rest and during Valsalva manoeuvre in the standard 2D cross sectional planes in 4 chambers and bicaval views $\left(0^{\circ}\right.$ and $95^{\circ}$, respectively) (Video 1 and Video 2 ); real-time 3D TE echocardiographic imaging during a second injection of contrast during Valsalva manoeuvre revealed passage of bubbles in the left atrium by both real-time 3D TE imaging (Figure 1 and Figure 2D; Video 3) and by the 2D sectioning in 
unconventional cross sectional planes allowed by 3D TE imaging intersecting the bubbles displayed on the 3D image (Figure $2 \mathrm{~A}-\mathrm{C}$; Video 4). It was possible to identify 16 bubbles. No other sources of embolism were detected.

\section{Discussion}

The management of PFO still remains controversial. According to the newest scientific evidences, the assessment of the PFO size and the shunt has paramount importance for further management [1]. Image the PFO is not easy, because of its complex anatomic morphology, the irregular shape of the opening and for the dynamic changes during the cardiac cycle [2-4]. Contrast transesophageal echocardiography provides detailed imaging of the interatrial septum and other relevant structures and the detection of the shunt itself [1-4]. Although 2D TEE contrast study has been considered to be the gold standard imaging modality for diagnosing PFO for a long time, new evidences underscore that its accuracy may have been over-valued $[1,5,6]$.

Indeed, a meta-analysis of the accuracy of TEE in the diagnosis of PFO compared to autopsy and cardiac surgery yielded a weighted sensitivity of only $89 \%[1,6]$. It is important to understand the limitations of 2D TEE and perhaps use other highly sensitive screening tests, such as transcranial doppler, in conjunction with 2D TEE before scheduling a patient for transcatheter PFO closure $[1,5,6]$. Inability to perform an adequate Valsalva manoeuvre during TEE is probably responsible but other factors can be involved [5,6]. In fact, the correct use of the Valsalva manoeuvre to provoke a right-to-left shunt is essential. A good quality of the Valsalva manoeuvre should

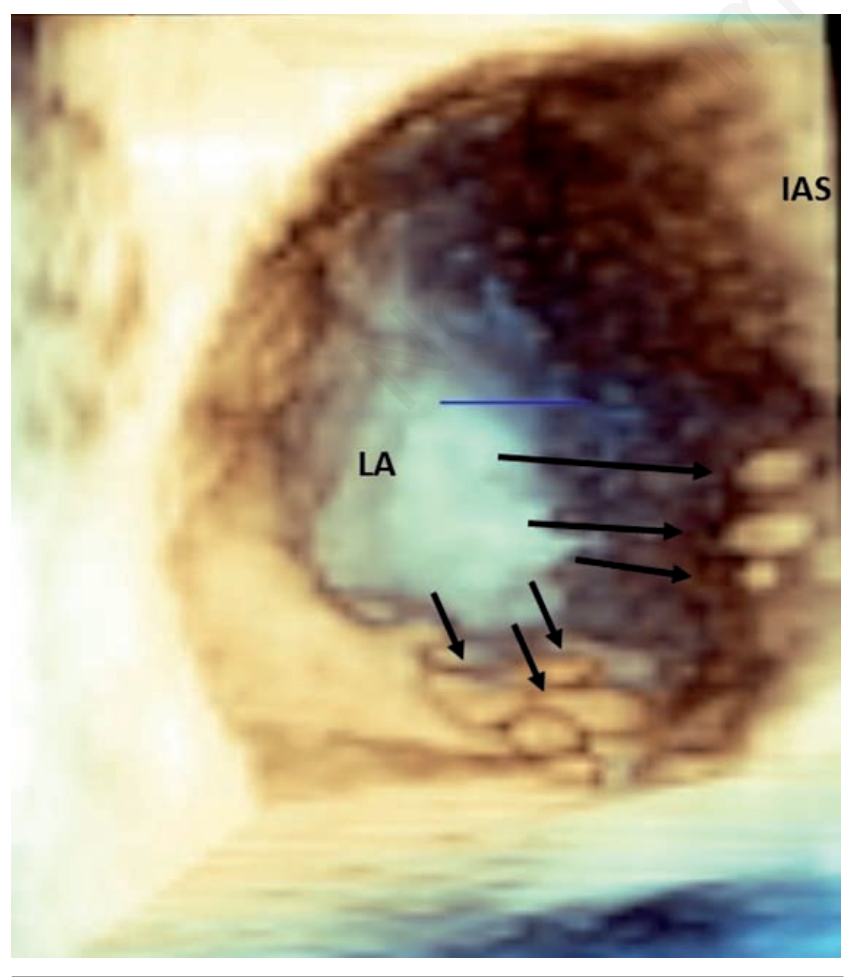

Figure 1. 3D TEE: 'en face' view from above of the left side of the interatrial septum after cropping of left atrial wall; black arrows point at the bubbles. LA, left atrium; IAS, interatrial septum. be considered effective by observing a $20 \mathrm{~cm} / \mathrm{sec}$ decrease in transmitral $\mathrm{E}$ velocity and a change in the convexity of the fossa ovalis membrane from right to left as a means of judging the effectiveness of the Valsalva manoeuvre creating sufficient intrathoracic pressure to decrease preload and sufficient to also increase the right atrial-toleft atrial pressure gradient $[5,6]$. Moreover, having in mind that the PFO has a 'mouth shaped' opening [3,4], that the bubbles can pass eccentrically across the PFO due to an asymmetrical PFO opening and that can be directed in any directions and that whole LA volume cannot be imaged by 2D TEE without using contemporary multiple cross sectional planes at the same time, we have to consider the possibility that by using only 2D TEE could lead to misdiagnose the presence and the numbers of the bubbles.

The advent and technological advances in 3D TEE imaging allow for the reconstruction of realistic anatomic images of cardiac structure and function $[3,4,7,8]$. Nowadays this imaging appears to be emerging as a diagnostic technique that overcomes some of the limitations of 2D E $[3,4,7,8]$. Currently, both transthoracic echocardiography and transesophageal echocardiography transducers may generate 3D images. However, 3D TEE, providing images of higher quality in comparison with 3D TT ECHO, is the only modality able to allow for a detailed anatomical imaging. 3D TEE allows an anatomical imaging for a comprehensive echocardiographic assessment of the left atrium, atrial septum and of PFO morphology $[3,4,7,8,9]$. Moreover, TEE 3D atrial septum image appears very detailed because the $3 \mathrm{D}$ system primarily uses the axial resolution to reconstruct the image that is superior to both lateral resolution and elevation (azimuth) resolution [3,4,7]. Furthermore, it permits the sectioning of the $3 \mathrm{D}$ volume by innumerable and unconventional $2 \mathrm{D}$ cross sectional planes oriented on the 3D image [7].

Currently, even though the low spatial and temporal resolution remains a fundamental limitation of real time 3D E, the platforms are able to achieve an excellent number of scan lines and the frame rates are also double than in the past and produces a superior image quality [9]. These technological advances in 3DE produce more accurate and reproducible measurements, mainly in the ventricular volume and ejection fraction, as recognized in the American Society of Echocardiography and European Association of Cardiovascular Imaging Chamber Quantification guidelines [10]. $3 \mathrm{D}$ TEE also produces reproducible imaging of the cardiac anatomy, in particular of the mitral valve and atrial septum.

Despite the advantages, the dissemination of 3DE imaging in clinical practice is slow, mostly because $3 \mathrm{D}$ imaging rely on a certain level of expertise and organization of the laboratory and the latest advances are only available in the latest version of the machines. However, its use is becoming routine in highly specialized centres for structural heart disease assessment and guidance of procedures, it is also becoming a key tool for communication between imaging specialists, general cardiologists, interventionists, and surgeons $[9,11]$.

In multimodality imaging scenarios, however, [magnetic resonance imaging (MRI)] and [computed tomography angiography (CTA)] have also been used to detect a PFO. Cardiac CT was used in a small number of patients with TEE identified PFO [12]. Using three different criteria (a distinct left atrial 'flap' representing the fossa ovalis, a continuous column of contrast connecting this flap to the right atrium, and a contrast jet traversing the column into the right atrium), they were able to identify four PFO patients $(67 \%)$ without using the Valsalva provocation manoeuvre [12]. In a recent study with 19 patients [13], the sensitivity and specificity of the CTA, compared with contrast TTE, in detecting the existence of a shunt were $53 \%(8 / 15)$ and $75 \%(3 / 4)$. The main limitations of this study were that the CTA was compared with TTE and not with 

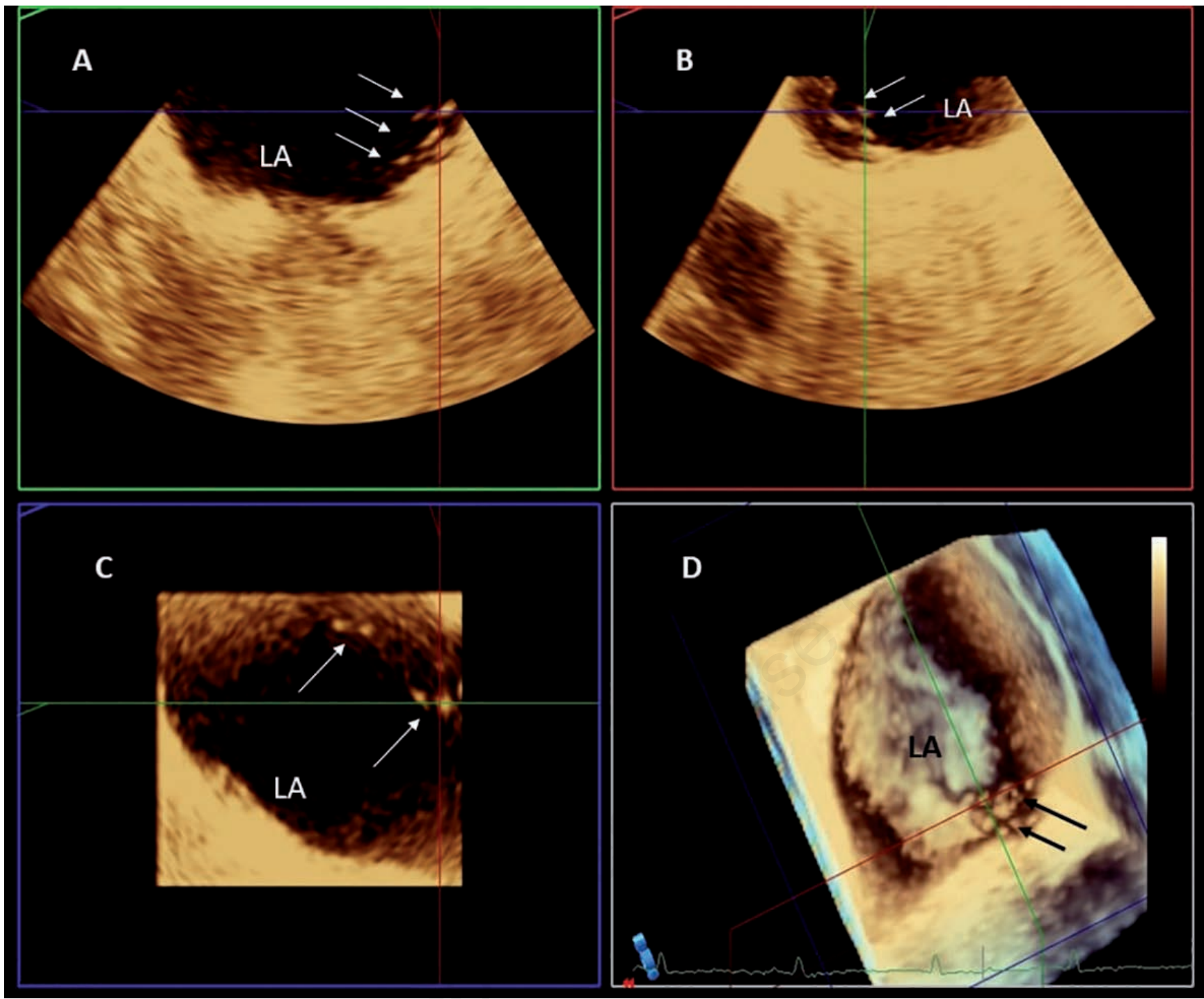

Figure 2. A-C) 2D sectioning in unconventional cross-sectional planes allowed by 3DE imaging intersecting the bubbles displayed on the 3D image; white arrows point at the bubbles. D) 3D TEE 'en face' view from above of the left side of the interatrial septum after cropping of left atrial wall showing the $2 \mathrm{D}$ electronical sectioning with planes intersecting the bubbles (black arrows). LA, left atrium.

TEE that is considered the gold standard. The CTA detected a leftto-right shunt while the echocardiography detected a right-to-left shunt and Valsalva manoeuvre cannot be performed in a CTA.

An MRI is another a non-invasive method to detect a PFO and atrial septal aneurysm. A study [14], with MRI compared with contrast-enhanced TEE for the detection of a PFO, had a sensitivity of $50 \%$ and a specificity of $100 \%$, a negative predictive value of $31 \%$, and a positive predictive value of $100 \%$.

The CTA and MRI are not recommended in the recent European Society of Cardiology consensus document for diagnosing a PFO [1]. Larger prospective studies are required to establish the definite role of cardiac CTA and MRI in the diagnosis of a PFO. Based on the currently available evidence, the two modalities should be regarded as complementary methods to echocardiography, providing additional information in specific situations; however, they are not recommended as first-line modalities.

It has never been reported about 3D TEE diagnostic usefulness in helping to detect and count the bubbles during contrast study. We report a case where it has been possible to detect the passage of bubbles across the PFO thanks to 3D TEE. In our report the lack of detection by $2 \mathrm{D}$ TEE may be explained by the fact that the PFO opening was asymmetric and that the bubbles were directed eccentrically to impinge interatrial septum and the atrial wall going in a plane not intersected by the standard cross sectional $2 \mathrm{D}$ planes. Moreover, we have also to consider technical factors as a not appropriate choice of the $2 \mathrm{D}$ cross sectional planes for imaging the PFO and a not correct use of the Valsalva manoeuvre to provoke a rightto-left shunt that are common potential sources of misdiagnose; however, it is doubtless that 3D TEE helped to diagnose the shunt.

The use of 3D TEE allowing en face view of the interatrial septum can help to confirm the presence and the number of the bubbles and to better guide and positioning the 2D TEE cross sectional planes. Having in mind that the numbers of bubbles has been correlated to the size of PFO and therefore engages subsequent therapeutic choices, a correct detection of the number of bubbles has paramount importance in identifying large size complex PFO. 


\section{Conclusions}

Even in the presence of only a report, our case highlights the mainstay importance of a careful 2D TEE assessment and suggests that nowadays, thanks to the latest advances, 3D TEE could have an additional value and may represent the key adjunctive echocardiographic technique being able to positively impact on the diagnosis and to compliment 2D imaging in PFO assessment. Further studies are needed to address the accuracy of 3DE in PFO assessment.

\section{References}

1. Pristipino C, Sievert H, D'Ascenzo F, et al. European position paper on the management of patients with patent foramen ovale. General approach and left circulation thromboembolism Eur Heart J 2019;40:1-14.

2. Mojadidi MK, Bogush N, Caceres JD, et al. Diagnostic accuracy of transesophageal echocardiogram for the detection of patent foramen ovale: A meta-analysis. Echocardiography 2014;31:752-8.

3. Rana BS, Shapiro LM, McCarthy KP, Ho SY. Three-dimensional imaging of the atrial septum and patent foramen ovale anatomy: defining the morphological phenotypes of patent foramen ovale. Eur J Echocardiogr 2010;11:i19-i25.

4. Faletra FF, Muzzarelli S, Dequarti MC, et al. Imaging-based right-atrial anatomy by computed tomography,magnetic resonance imaging, and three-dimensional transoesophageal echocardiography: correlations with anatomic specimens. Eur Heart J Cardiovasc Imaging 2013;14:1123-31.

5. Rodrigues AC, Picard MH, Carbone A, et al. Importance of adequately performed Valsalva maneuver to detect patent foramen ovale during transesophageal echocardiography. J Am Soc Echocardiogr 2013;26:1337-43.
6. Johansson MC, Eriksson P, Guron CW, Dellborg M. Pitfalls in diagnosing PFO: Characteristics of false-negative contrast injections during transesophageal echocardiography in patients with patent foramen ovale. J Am Soc Echocardiogr 2010;23:1136-42.

7. Lang RM, Badano LP, Tsang $\mathrm{W}$ et al. EAE/ASE recommendations for image acquisition and display using three-dimensional echocardiography. Eur Heart J Cardiovasc Imaging 2012;13:1-46.

8. Aggeli C, Verveniotis A, Andrikopoulou E, et al. Echocardiographic features of PFOs and paradoxical embolism: a complicated puzzle. Int $\mathrm{J}$ Cardiovasc Imaging 2018;34:1849-61.

9. Lang RM, Addetia K, Narang A, Mor-Avi V. 3-Dimensional Echocardiography: Latest Developments and Future Directions. JACC Cardiovasc Imaging 2018;11:1854-78.

10. Lang RM, Badano LP, Mor-Avi V, et al. Recommendations for cardiac chamber quantification by echocardiography in adults: an update from the American Society of Echocardiography and the European Association of Cardiovascular Imaging. J Am Soc Echocardiogr 2015;28:1-39.

11. Galzerano D, Kinsara AJ, Di Michele S, et al. Three dimensional transesophageal echocardiography: a missing link in infective endocarditis imaging?. Int J Cardiovasc Imaging 2020;36:403-13.

12. Williamson EE, Kirsch J, Araoz PA, et al. ECG-gated cardiac CT angiography using 64-MDCT for detection of patent foramen ovale. AJR Am J Roentgenol 2008;190:929-33.

13. Kara K, Sivrioğlu AK, Öztürk E, et al. The role of coronary CT angiography in diagnosis of patent foramen ovale. Diagn Interv Radiol 2016;22:341-6.

14. Hamilton-Craig C, Sestito A, Natale L, et al. Contrast transoesophageal echocardiography remains superior to contrastenhanced cardiac magnetic resonance imaging for the diagnosis of patent foramen ovale. Eur J Echocardiogr 2011; $12: 222-7$

\section{VIDEO LEGENDS}

Video 1. 2D TEE; bicaval view at $95^{\circ}$; no bubbles seen in the left atrium.

Video 2. 2D TEE; 4 chambers view at 00 ; no bubbles seen in the left atrium.

Video 3. 3D TEE; en face left atrial side of the interatrial septum view; imagine of bubbles.

Video 4. 3DE 2D electronical sectioning with planes intersecting the bubbles. 